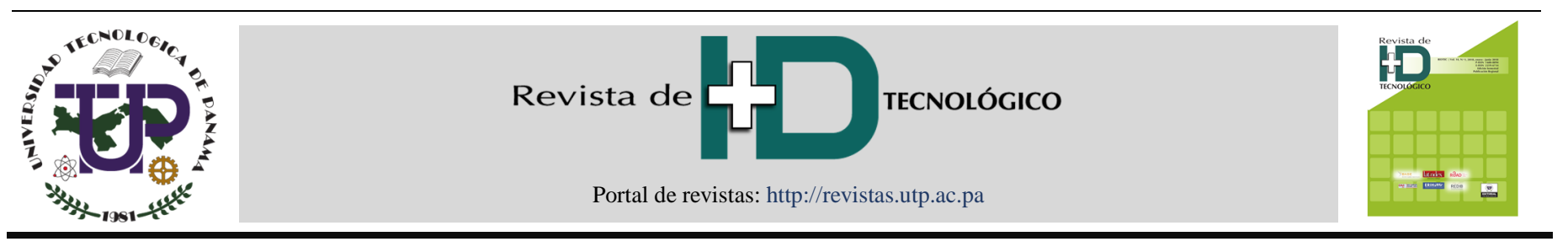

\title{
Estado del Arte de conmutación de líneas de transmisión con análisis de contingencias
}

\section{State of Art transmission systems switching with contingency analysis}

\author{
Paúl Andrés Masache Almeida ${ }^{1 *}$, Diego Francisco Carrión Galarza ${ }^{2}$ \\ 1, 2 Universidad Politecnica Salesiana, Ecuador \\ *Autor de correspondencia: pmasache@ups.edu.ec
}

RESUMEN- La presente investigación está enfocada en óptima conmutación de líneas de transmisión (OTS) posterior a la presencia de contingencias N-1 en los sistemas de potencia; por medio de OTS se reconfigura la etapa de transmisión tras producirse una falla, de esta manera; se busca disminuir la pérdida o salida de demanda del sistema y de reducir la probabilidad de un apagón masivo. OTS es un método muy flexible que permite solventar varios problemas que se presentan en el sistema eléctrico; principalmente en pérdidas y costos operativos. Se plantean las ecuaciones que rigen al OTS y una aproximación del método; el objetivo del uso de OTS es de mantener en los márgenes mínimos de operación al sistema eléctrico (tensión, frecuencia, el balance de potencias entre la etapa de generación y la demanda), frente a una contingencia N-1 y se evaluará la confiabilidad del SEP tanto en prefalla, como en postfalla por medio de los índices de confiabilidad. OTS es una metodología que permite reestructurar la etapa de transmisión del SEP, para mantener los niveles adecuados al sistema y reducir las pérdidas que pueden producirse.

Palabras clave - Análisis de contingencias, conmutación de líneas de transmisión, índices de confiabilidad, sistema eléctrico de potencia, sistema de transmisión.

\begin{abstract}
The next investigation is focused in optimal transmission line switching (OTS) after presence of N-1 contingency in electrical power system; OTS chance topology transmission system when in the system is produced a fault, in this manner; this methodology search reduce the losses or demand output of the grid, however the principal idea is reducing the probability of blackout. OTS is a flexible method that permit to solve a lot of problems in the Electrical System. The principal problems are losses and operative costs. In this State of art, we detail the OTS equations and a method approximation. The principal idea is keeping the parameters in the minimum operations in Electrical System (voltage, frequency, generation and demand balance), with N-1 contingency; in SEP evaluate the reliability in prefault and postfault. we use reliability indices to evaluate SEP. OTS method, we permit to do a dynamic restructuring of SEP, and the method permit to keep the parameters and reduce the losses in the system, cause by faults.
\end{abstract}

Keywords-Contingency analysis, transmission line switching, reliability indices, electrical power system, transmission system.

\section{Introducción}

El sistema eléctrico de potencia (SEP), está conformado por tres Etapas: generación, transmisión y distribución; En la etapa de distribución se encuentra conectada toda la demanda que la etapa de generación debe abastecer; pero el punto de enlace entre las etapas de distribución y generación, es la etapa de transmisión; la etapa de transmisión se encarga de transportar la energía eléctrica producida por la etapa de generación para que esta energía sea distribuida por todo los consumidores finales; es decir, transporta la energía desde la etapa de generación hasta la distribución. Para diseñar la etapa de transmisión es necesario conocer la demanda que requiere el consumidor, de acuerdo a la demanda se establece los límites de potencia que soporta la etapa de transmisión y dependiendo donde se encuentre ubicada la etapa de generación se diseña la topología de la etapa de transmisión para transmitir la energía eléctrica desde etapa de generación hasta la distribución; la topología del sistema, se va expandiendo dependiendo del crecimiento de la etapa de demanda; mientras mayor incremento de cargas, la planificación de la expansión del sistema aumenta, sin embargo, mientras no exista una adecuada expansión del sistema, los estudios que se realizan o las simulaciones son fundamentales para la estabilidad de sistema estático [1].

Citación: P. Masache y D. Carrión, "Estado del Arte de conmutación de líneas de transmisión con análisis de contingencias," Revista de I+D Tecnológico, vol. 15, no. 2, pp. (98-106), 2019.

Tipo de artículo: Original. Recibido: 7 de marzo de 2019. Recibido con correcciones: 19 de junio de 2019. Aceptado: 26 de julio de 2019.

DOI. https://doi.org/10.33412/idt.v15.2.2252

Copyright: 2019 P. Masache y D. Carrión. This is an open access article under the CC BY-NC-SA 4.0 license (https://creativecommons.org/licenses/by-nc$\mathrm{sa} / 4.0 /)$. 
Pero en la actualidad es necesario considerar al sistema de manera dinámico, es decir, que la etapa de demanda no es una variable constante, y viene a ser una variable dinámica; por la razón, es necesario realizar estudios del comportamiento de la etapa de demanda, en conjunto con el crecimiento de la etapa de generación. La metodología de la óptima conmutación de líneas de transmisión (OTS) que proviene de la terminología inglesa optimal transmission switching, permite realizar una planificación de la etapa de transmisión dependiendo del comportamiento de la etapa de la demanda y así optimizar el uso de las fuentes de energía eléctrica [1][2]; también OTS permite realizar la conmutación de las líneas de transmisión en caso de perturbaciones eléctricas y en conjunto con estudios de análisis de contingencias se conoce el nivel de seguridad que posea el sistema; es decir, la confiabilidad.

Se plantea usar la óptima conmutación de líneas de transmisión (OTS) que permite realizar la conmutación de las etapa de transmisión, para mantener los parámetros principales de un sistema eléctrico como son: las caídas de tensión, sobrecarga de líneas, balance de potencia entre demanda que requiere el consumidor y la etapa de generación [3]. Existen diferentes problemas que se pueden resolver con el modelo OTS como por ejemplo: la planeación de la expansión del sistema considerando que tiene un comportamiento dinámico [4], seguridad, confiabilidad del sistema, reducción del costo de producción de energía [5][6], disminución de la sobrecarga de las líneas [7], uno de los puntos fundamentales de OTS es la conmutación de las líneas cuando existen fallas eléctricas, para mantener al sistema bajo los parámetros óptimos de operación (voltaje, frecuencia, satisfacer la demanda) [8][9][10].

En este artículo se hace énfasis en el uso del OTS para la conmutación de las líneas en caso de producirse una falla eléctrica y, por medio de un análisis de contingencias, principalmente un estudio para una contingencia N-1 y verificar la confiabilidad del mismo antes y después de la conmutación; una contingencia es cuando un elemento del sistema sale de operación por una falla [11]; las fallas que pueden afectar al SEP pueden ser; la salida de grandes cargas de manera esporádica y las perturbaciones que se producen en las barras del SEP, este tipo de contingencias afectan directamente a la etapa de generación y distribución.
Existen varios métodos que se pueden usar para realizar un análisis de fallas del sistema, por ejemplo: el flujo de potencia y flujo óptimo de potencia AC y, dependiendo de la complejidad el flujo de potencia y flujo óptimo de potencia DC [12][13][14][15].

La confiabilidad permite comprobar el nivel de seguridad que posee el sistema cuando se produce una falla, las fallas alteran los parámetros de operación, la finalidad de un estudio de confiabilidad es conocer si el sistema es capaz de soportar una contingencia N-1, NM. También es conocer aquellos elementos vulnerables; para ello se emplean los índices de confiabilidad y así tener una mejor comprensión del comportamiento del sistema. Cuando se habla del uso del método OTS, es restructurar la transmisión manteniendo los parámetros de operación y principalmente que no afecte el balance de potencia [12][16].

Las principales contribuciones que presenta este artículo son: un enfoque más amplio del uso OTS junto a un análisis de contingencias; OTS como modelo base para el comportamiento de la etapa de transmisión frente a una falla eléctrica y cómo se reestructura; planteamiento de las expresiones matemáticas que representan al OTS; las aproximaciones realizadas con respecto del OTS por [2] y los teoremas fundamentales que gobiernan a estas aproximaciones frente al método OTS original.

En síntesis, el método OTS tiene una gran flexibilidad para la solución de varios problemas; varios autores han propuesto la solución de: Estabilidad de voltaje, sobrecarga de las líneas eléctricas, reducción de pérdidas y costos operativos, seguridad en el sistema de potencia, análisis de conmutación de líneas durante una emergencia [17][18][19], protecciones en el sistema eléctrico y la reestructuración dinámica (Topología dinámica) [20][21]. A continuación, se detalla cómo está estructurado el articulo; sección II, Aplicaciones del OTS; sección III, Análisis de contingencias, sección IV, Expresiones matemáticas del OTS; sección V, Índices de Confiabilidad; sección VI, Conclusiones y Futuros Trabajos.

\section{Aplicaciones de la conmutación de líneas de transmisión}

Existen varios aplicativos de la conmutación de líneas; la principal ventaja es optimizar el uso de los recursos y mantenimiento al sistema operativo frente a 
diversas contingencias o frente a una expansión del sistema.

Cuando se habla de la expansión del sistema, existen algunas variables que se debe considerar al momento de planificar una expansión; uno de los puntos principales de interés es el factor económico, ya que se debe equilibrar entre la inversión y la posibilidad de integrar nuevas cargas de demanda al sistema; otro aspecto fundamental es la solución del problema de congestión en las líneas eléctricas [22], cuando se habla de reducir la congestión de las líneas existen dos maneras como solucionar el problema, redefinir al sistema, es decir cambiar la topología del sistema o implementar nuevas estructuras en el sistema; muchos autores proponen realizar la planificación de la expansión por medio de la metodología expuesta OTS; el modelo OTS realiza una expansión del sistema eléctrico a corto y mediano plazo; cuando se realiza un análisis de expansión, OTS reestructura, rediseña la etapa nueva de transmisión de acuerdo a parámetros técnicos eléctricos y como se ha consolidado la etapa de distribución y se verifica la confiabilidad de la nueva topología. [23], [24].

También por medio del OTS se realiza la conmutación o desconexión de líneas en caso de alguna emergencia cuando existe una falla en el sistema; como se conoce una falla altera la corriente y el nivel de tensión, esto provoca que el sistema pierda estabilidad y en caso de que la falla persista, puede provocar la perdida de la etapa de demanda; OTS es una metodología que permite redistribuir el flujo de corriente eléctrica por medio de la desconexión de las líneas y de esta manera minimizar la falla producida en el sistema eléctrico, el punto importante de realizar un OTS cuando se produce una falla eléctrica es mínima la desconexión de carga, con la finalidad de que el consumidor no se vea afectado la consumo habitual de energía eléctrica [1], [2].

El principal aspecto del OTS a tomar en cuenta, es la capacidad que tiene para aportar la etapa de generación, la potencia que necesita la etapa de demanda, una reserva en caso de emergencia; en cambio para la etapa de transmisión se debe tener en cuenta lo generado y la capacidad para transmitir toda la potencia que requiera la etapa de demanda, otro aspecto es la estabilidad que posea con respecto a una perturbación, y que esta perturbación tenga una mínima influencia en el funcionamiento del sistema. La etapa de distribución es la capacidad de suministrar la energía proporcionada por la etapa de trasmisión a cada una de las grandes cargas; para ello el sistema debe estar dividido en circuitos primarios de alimentación, el nivel de seguridad y protección que posea las subestaciones [25], [26].

El modelo OTS es uno de los medios que permite solventar varias problemáticas que se plantean en el sistema de potencias, por tal razón se realiza una profundización en cuanto a la metodología que se plantea y a los 3 principales teoremas que gobiernan a este método planteado por [2]; además existen varios autores que realizan la restructuración del sistema por el OTS aplicando flujo de carga DC [27][7] y un análisis por flujo de carga AC [12][27][5]. El análisis principalmente se lo realiza bajo los parámetros de voltajes del sistema [11][16][28]. El tipo de problema que se plantea es MILP (programación lineal entera mixta), con variables binarias; las variables binarias representaran el estado de operación de las líneas, también es necesario el uso de un Flujo de Potencia Optimo para verificar el comportamiento del SEP antes y después de la perturbación [29], [30], [31]; por medio de un flujo óptimo de carga se obtendrá los niveles de potencia que se genera para abastecer la etapa de demanda, por ultimo con el OTS se obtiene la topología de la etapa de transmisión después de la perturbación [32][33]. La finalidad es mantener la estabilidad del sistema, reduciendo el uso de los recursos; otro factor importante del método OTS es reducir el costo operativo cuando el sistema presenta fallas inesperadas, manteniendo niveles de tensión y frecuencia de manera óptima y satisfaciendo el balance de potencias (la potencia generada será igual a la demanda necesaria más las pérdidas producidas en el sistema) [34][35].

\section{Análisis de contingencias}

El análisis de contingencias se puede realizar ya sea en la etapa de generación, transmisión o distribución; pero para el estudio planteado se realiza únicamente en la etapa de transmisión; la finalidad de realizar análisis es que después de una perturbación el sistema sea capaz de soportar y pueda mantener su operación bajo condiciones mínimas de operación [6][36][37]. Dicho análisis es una parte fundamental del estudio de la seguridad que puede brindar el sistema eléctrico; una contingencia es cuando un elemento sale de servicio o 
deja de operar; el análisis ayuda a conocer el comportamiento del sistema cuando no opera en condiciones normales [7], por ejemplo, cuando un generador no funciona, un cortocircuito eléctrico o una pérdida significativa de demanda eléctrica [16][22]. La principal salida de elementos del sistema es por fallas, y esta falla puede producir salidas de un solo elemento o puede provocar la salida de múltiples elementos.

Un análisis de contingencias se lo efectúa con la finalidad de conocer el estado del sistema antes de que se produzca la falla y después de la falla, para conocer el estado del sistema y tener claro que elementos pueden operar bajo condiciones anormales; además, es un parámetro fundamental cuando se realiza un estudios del sistema eléctrico [6]. Generalmente el estudio se lo efectúa en un análisis del tipo $\mathrm{N}-1$ [27]; una contingencia $\mathrm{N}-1$, hace referencia a la capacidad del SEP de reaccionar frente a un elemento en falla, pero principalmente es la habilidad de los generadores de estabilizar al sistema frente a esta falla [12]. Un análisis de contingencias posee parámetros fundamentales de operación como es la tasa de falla y la tasa de reparación; los dos parámetros son la base del cálculo de los índices de confiabilidad:

La confiabilidad del SEP es verificar si el sistema es capaz de soportar una falla y conocer el nivel de seguridad, manteniendo protegido el sistema; existen índices que permite cuantificar y medir al sistema; pero como el SEP es muy amplio se lo ha dividido en tres etapas: generación, transmisión y distribución [25].

La tasa de falla (TTF), es un valor que se lo calcula a partir del número de interrupciones del servicio que ha sufrido un elemento del sistema; cada elemento del sistema posee un valor de tasa de falla (TTF), a este índice o valor se lo calcula en un tiempo determinado. El valor inverso de la tasa de fallas se lo conoce como tiempo promedio entre fallas (MTTF) $y, U$ es la indisponibilidad anual de un equipo [25].

$$
T T F=M T T F * \ln (U)
$$

La tasa de reparación (TTR), se le conoce como el tiempo estimado en realizar la reparación del elemento en falla; de manera técnica a este valor se lo conoce también como el tiempo promedio que puede durar una falla; el valor inverso de la tasa de reparación se lo conoce como tiempo promedio de reparación (MTTR) $\mathrm{y}, \mathrm{U}$ es la indisponibilidad anual de un equipo [25].

$$
T T R=M T T R * \ln (U)
$$

La confiabilidad del SEP está determinada por las fallas que pueden producirse durante un tiempo determinado, muchos autores mencionan que la confiabilidad se cuantifica en un lapso promedio de 5 minutos; es decir, que durante este tiempo el SEP debe estabilizar sus parámetros de operación (voltaje, frecuencia y el balance de potencias); por ende, la confiablidad solo estudia la frecuencia y la duración de la falla. Cuando el sistema no se estabiliza durante el tiempo esperado, se produce un colapso general de operación y control del sistema. Se necesita que el sistema sea capaz de recuperarse rápidamente de los fallos, esta capacidad de recuperación se lo conoce como resiliencia [38][39]; la resiliencia posee varias etapas o condiciones para su operación; primero la protección necesaria para operar bajo condición anormal, segundo la redundancia; es decir, una reserva como resguardo en caso de daño mecánico y tercero la recuperación del sistema de manera temprana; por tal razón, un sistema tiene que tener la habilidad de recuperarse y adaptarse frente a fallos [38][40].

En la sección VI se realiza un detalle sobre los índices que se pueden usar en cada una de las etapas descritas.

\section{Expresiones matemáticas del OTS.}

Las expresiones matemáticas que se detallan son desarrolladas por [2], y la base teórica de este desarrollo se realizó en [1]; por tal razón se menciona un detalle matemático expuesto por el autor [2], y todo el análisis del OTS está fundamentado o es una extensión matemática de un flujo óptimo de carga DC.

\section{Nomenclatura.}

$\begin{array}{ll}n & \text { Número de generadores } \\ k & \text { Línea de transmisión } \\ i & \text { Número de bus } \\ C_{n} & \text { Costo de operación de un generador } \\ D_{b} & \text { Demanda eléctrica } \\ F_{k}{ }^{m a ́ x} & \text { Máxima tasa de la línea de transmisión } \\ Y_{k} & \begin{array}{l}\text { Susceptancia eléctrica de la línea de } \\ \text { transmisión }\end{array}\end{array}$




$\begin{array}{ll}G_{n}{ }^{\text {máx }} & \text { Generación máxima } \\ G_{n}^{\text {min }} & \text { Generación mínima } \\ \theta^{\text {min }} & \text { Ángulo mínimo } \\ \theta^{\text {máx }} & \text { Ángulo máximo } \\ \theta_{a} & \text { Ángulo de origen del bus } \\ \theta_{b} & \text { Ángulo de destino del bus } \\ Z_{k}{ }^{\text {máx }} & \text { Número máximo de líneas conmutadas } \\ Z_{k} & \begin{array}{l}\text { Estado de la línea (0 fuera de servicio; } \\ \text { 1 en servicio) }\end{array} \\ g_{n} & \text { Potencia del generador } \\ f_{k} & \begin{array}{l}\text { Flujo de potencia transmitido por la } \\ \text { línea }\end{array} \\ M_{k} & \text { Variable para optimizar } \\ & \text { Valor Máximo de Potencia de las }\end{array}$

Las expresiones matemáticas mostradas en las ecuaciones (3-9) son todas las que representan matemáticamente el modelo OTS.

Función Objetivo:

$$
\text { Min: } P=\sum C_{n} * g_{n}
$$

s.a.

$$
\begin{gathered}
\sum g_{n}+\sum f_{k}-\sum f_{k}=D_{i} \quad \forall b \\
-\left(1-z_{k}\right) M_{k} \leq f_{k}-Y_{k}\left(\theta_{a_{k}}-\theta_{b_{k}}\right) \\
\leq\left(1-z_{k}\right) M_{k} \quad \forall k \\
-F_{k}^{\text {max }} z_{k} \leq f_{k} \leq F_{k}^{\max } z_{k} \quad \forall k \\
G_{n}^{\text {min }} \leq g_{n} \leq G_{n}^{\max } \quad \forall n \\
-\theta_{k}^{\max } \leq \theta_{a_{k}}-\theta_{b_{k}} \leq \Theta_{k}^{\max } \quad \forall k \\
\sum\left(1-z_{k}\right)=Z_{k}^{\text {max }}
\end{gathered}
$$

La ecuación (3), representa la función objetivo, minimizar la función de costos de operación por la potencia que necesita el generador despachar; la ecuaciones de (4-9) representan todas las restricciones a las cuales está sujeto el modelo; la ecuación (4) figura la sumatoria de potencia generada con la diferencia del flujo de potencia del bus y es igual a la potencia que sale de la misma barra; la ecuación (5) manifiesta la relación que existe entre los parámetros de voltaje y ángulo entre los buses y las líneas que se interconectan a los buses; la ecuación (6) representa los límites de flujo que posee una línea; la ecuación (7) traza la capacidad máxima y mínima de cada generador; la ecuación (8) restringe la diferencia entre los ángulos de los buses, forzándolo entre un valor mínimo y un valor máximo. En las restricciones mostradas, también se incluye la conmutación de las líneas, el termino $Z_{\mathrm{k}}$, es una variable binaria que representa el estado de operación de la líneas eléctricas [2].

\section{Metodología aproximada del OTS}

Las expresiones matemáticas de las ecuaciones (3-9), representan las ecuaciones originales del OTS estas ecuaciones son propuestas por [1], pero también se presenta un modelo aproximado del OTS, en donde se incluye una variable de decisión $\epsilon_{\mathrm{k}}$, y una contante en la función objetivo C'; esta aproximación es propuesto por [2].

Función Objetivo:

$$
\text { Min: } P=\sum C_{n} * g_{n}+C^{\prime} \sum \frac{\epsilon_{k}}{M_{k}}
$$

s.a.

$$
\begin{gathered}
\sum g_{n}+\sum f_{k}-\sum f_{k}=D_{i} \quad \forall b \\
-\epsilon_{k} \leq f_{k}-Y_{k}\left(\theta_{a_{k}}-\theta_{b_{k}}\right) \leq \epsilon_{k} \quad \forall k \\
0 \leq \epsilon_{k} \leq\left(1-z_{k}\right) M_{k} \quad \forall k \\
-F_{k}^{\max } z_{k} \leq f_{k} \leq F_{k}^{\max } z_{k} \quad \forall k \\
G_{n}^{\min } \leq g_{n} \leq G_{n}^{\max } \quad \forall n \\
-\Theta_{k}^{\text {max }} \leq \theta_{a_{k}}-\theta_{b_{k}} \leq \Theta_{k}^{\max } \quad \forall k
\end{gathered}
$$

Al igual que las expresiones del OTS en las ecuaciones (3-9), la formulación matemática presentada en las ecuaciones (10-16), posee una función objetivo la ecuación (10) donde se minimiza la función de costos de operación junto a la potencia adecuada que debe producir cada generador; las ecuaciones $(11-16)$ son restricciones, la ecuación (11) representa el flujo entre los buses con la sumatoria de la generación requerida; la ecuación (5) es la relación entre el flujo de potencia junto al ángulo de desfase que va a tener cada bus; las ecuaciones (14-16) representan los valores máximos y mínimos que van a restringir al flujo de potencia, a la producción de potencia de cada generador, la variación 
angular permitida en cada bus. En las ecuaciones del (12-16) interviene una variable binaria $\epsilon_{\mathrm{k}}$ que puede tomar valores de cero o uno dependiendo del estado de las líneas, y la C' es una constante del sistema. Se plantea tres teoremas que permiten una mejor comprensión a la metodología del OTS [2].

Teorema 1. Las soluciones óptimas encontradas con el OTS original son similares a las soluciones óptimas encontradas con el modelo del OTS aproximado [2].

Teorema 2. Si se asume que "a1" y "a3" es número de líneas conmutadas, ya sea por la metodología original del OTS o por la metodología aproximada del OTS; estos valores realizan el remplazo en la (12); entonces se ha obtenido la siguiente relación como resultado [2]:

$$
a 1 \leq a 3
$$

Teorema 3. Se asume "a" es el número de líneas conmutadas como solución óptima de la metodología del OTS original y también de la metodología del OTS aproximado, por lo tanto, se concluye la siguiente relación entre las dos funciones objetivo [2].

P_3-P_1 $\leq \mathrm{a}$ Para la F.O. del OTS original

P_3-P_1 $\leq$ C'*a Para la F.O del OTS aproximado

\section{6. Índices de confiabilidad}

Los índices de la calidad del suministro de energía, permite establecer las afectaciones que se tuvieron durante un corte de suministro, cuantas veces se produjeron estos fallos; a estos indicadores se puede evaluar en la etapa de generación, la etapa de transmisión, y la etapa de distribución de electricidad.

ASAI (Avarage Service Avialability Index); número de horas disponibles vs el número de horas que se ha brindado el servicio; se usa en la etapa de distribución [41][42].

$$
A S A I=\frac{\text { disponibilidad de servicio }}{\text { total de horas de servicio }}
$$

ASIDI (Avarage System Interruption Duration Index); tiempo en donde se detuvo en su totalidad el servicio de energía, se usa en la etapa de distribución [41][43].

$$
A S A D I=\frac{\text { total de KVA no suministrado }}{K V A \text { suministrado }}
$$

ASIFI (Average System Interruption Frecuency Index); número total de interrupciones que se ha generado por un periodo de tiempo; se relaciona con una interrupción del servicio global o una interrupción del servicio parcial; se usa en la etapa de distribución [41].

$$
A S I F I=\frac{\text { KVA no suministrado }}{\text { KVA suministrado }}
$$

ASUI (Average Service Unavailability); número de desconexiones que ha tenido un elemento del sistema, es decir el número de veces que ha dejado de brindar el servicio eléctrico a los clientes durante un periodo de tiempo determinado; se usa en la etapa de distribución. En síntesis, este índice significa el servicio medio que no está disponible [42].

$$
A S U I=1-A S A I
$$

CAIDI; número total de fallos que ha existido en un año, no son fallos recurrentes o fallos en un solo equipo; representa los fallos globales en el sistema, se utiliza en la etapa de distribución [41][42][43].

$$
\text { CAIDI }=\frac{\text { minuto } \text { sin servicio por cliente }}{\text { total de clientes sin servicio }}
$$

CAIFI; se utiliza en la etapa de distribución, permite medir el número de interrupciones en el año de un consumidor [41].

$$
\text { CAIFI }=\frac{\text { número de veces } \sin \text { servicio }}{\text { total de clientes sin servicio }}
$$

MAIFI; valor medio de las veces que un equipo ha salido de operación, que ha dejado de suministrar energía eléctrica durante un periodo de un año [41].

$$
\text { MAIFI }=\frac{\begin{array}{c}
\text { número total de interrupciones del } \\
\text { cliente menos el tiempo definido }
\end{array}}{\text { total de clientes sin servicio }}
$$

SAIDI; tiempo de duración de una falla, en donde está localizada la falla y los recursos disponibles para solventar la falla; se utiliza en la etapa de distribución [41][42].

$$
S A I D I=\frac{\text { minutos } \text { sin servicio por cliente }}{\text { total de clientes }}
$$

SAIFI; frecuencia de interrupción de una falla; es decir, cuántas veces se ha producido la falla durante un 
periodo indefinido. Se utiliza en la etapa de distribución [41][42].

$$
\text { SAIFI }=\frac{\text { total clientes sin servicio }}{\text { total de clientes }}
$$

LOLE; número de horas que se conoce o se sabe que no se abastecerá la demanda; hace más referencia a mantenimientos ya planificados; se utiliza en etapa de generación, transmisión [44][45].

$$
L O L E=\sum T_{k} * P_{k}
$$

De donde $\mathrm{P}_{\mathrm{k}}$ es la probabilidad de la capacidad de suministrar la energía; $T_{k}$ es la demanda conocida no suministrada.

EENS; es un índice probabilístico, para evaluar la confiabilidad del sistema eléctrico y su evolución; representa a la energía no suministrada, principalmente durante un periodo de un año [44].

$$
\text { EENS }=\sum E_{k} * P_{k}
$$

De donde $\mathrm{P}_{\mathrm{k}}$ es la probabilidad de la capacidad de suministrar la energía; $E_{\mathrm{k}}$ es la energía no suministrada.

EUE (Expected Unserved Energy); este índice es la energía que se espera que no estará disponible en un periodo determinado [42].

$$
E U E=\sum L * U
$$

Donde L es la carga promedio conectada y la U es la indisponibilidad promedio anual del servicio.

LOEE; se utiliza en la etapa de generación y transmisión, representa la energía que no se suministra por la falta de capacidad de la infraestructura [45][46].

$$
L O E E=\sum E_{k} * p_{k}
$$

De donde $\rho_{\mathrm{k}}$ es la probabilidad de que la etapa de generación no abastezca la demanda; $E_{k}$ es la energía que no se puede suministrar.

LOLP (Loss Of Load Probability); representa el número de horas en el año, donde no se abasteció la demanda en totalidad; es utilizado en la etapa de generación, transmisión y en distribución en caso de generación distribuida [45].

$$
L O L P=\frac{L O L E}{\text { horas no suministradas }}
$$

EIU (Energy Index Unreliability); tasa de la energía del sistema que no se toma en cuenta cuando existe un inconveniente en el sistema, es energía no disponible [46].

$$
E I U=\frac{L O E E}{E}
$$

Los últimos índices no son aplicables cuando existe una reducción o un incremento de la demanda, se utiliza en la etapa de transmisión y a la etapa de generación, aunque, principalmente se utiliza en mantenimientos programados [46][47].

\section{Conclusiones}

Con la expansión del SEP, es necesario buscar formas de mantener al sistema operativo sin alterar los parámetros óptimos de funcionamiento; por tales razones se encuentra en estudio el método OST, optima conmutación de la etapa de transmisión, es un método que ha permitido realizar una reestructuración, variando su estructura, dependiendo de los niveles de demanda y de la caída de tensión de cada uno de los nodos del sistema.

OTS es un método que permite realizar diferentes tipos de estudios y mejorar la funcionalidad del SEP como, por ejemplo, el mejoramiento de los parámetros operativos del sistema; reduciendo las pérdidas producidas por la sobrecarga de las líneas.

La reestructura de la etapa de transmisión es la base de la metodología del OTS, los primeros estudios realizados se basan en una reconfiguración, pero a partir de estas bases de la investigación se fundamenta todas las aplicaciones y toda la propuesta; pero para ello es necesario incorporar otros fundamentos matemáticos como son los flujos de óptimo de potencia en DC o en AC y también, es necesario el uso de un despacho óptimo de generación. Por todo lo mencionado se considera que el problema se puede solucionar por medio de MILP.

El análisis de contingencias ayuda a conocer el comportamiento de un sistema cuando se encuentra sometido a una falla inesperada, cómo el sistema puede mantenerse operativo y cuáles son las condiciones de posfalla que se encontrará en el SEP. Por medio del OTS se propone realizar una conmutación de las líneas en caso de una falla, y por medio de esta reestructuración topológica buscar la mejor topología para aislar la falla y mantener el servicio eléctrico en las condiciones óptimas de operación (tensión, frecuencia constante y el balance de potencias, potencia generada sea igual a la demanda y las pérdidas del sistema). 


\section{Futuros trabajos}

El presente artículo es una premisa de una investigación realizada con respecto al modelo OTS expuesto; se pretende utilizar OTS en la etapa de transmisión para realizar una óptima conmutación de las líneas en caso de fallas en el sistema eléctrico, también se verifica el nivel de confiabilidad del SEP antes de la falla y, durante la falla por medio de los índices de confiabilidad (antes y después de la conmutación de las líneas); para concluir, se verifica el comportamiento del sistema durante la falla y se reestablece su operación por la metodología OTS a partir del modelamiento y la simulación, así se comprueba que el sistema eléctrico opere de manera adecuada después de la falla producida en el sistema.

\section{Referencias}

[1] E. B. Fisher, R. P. O'Neill, and M. C. Ferris, "Optimal Transmission Switching," IEEE Trans. power Syst., vol. 23, no. 3, pp. 1346-1355, 2008.

[2] M. Jabarnejad, "Approximate optimal transmission switching," Electr. Power Syst. Res., vol. 161, pp. 1-7, 2018.

[3] A. S. Korad and K. W. Hedman, "Reliability and stability analysis of corrective topology control actions," 2015 IEEE Eindhoven PowerTech, PowerTech 2015, 2015.

[4] A. K. Gupta, D. Kiran, and A. R. Abhyankar, "Flexibility in transmission switching for congestion management," 2016 Natl. Power Syst. Conf. NPSC 2016, 2017.

[5] S. Gusev and V. Oboskalov, "Recursion Based Contingency Analysis of an Electrical Power System," Int. Symp. Ind. Electron., pp. 2-5, 2016.

[6] M. Chen, "Dynamic Contingency Re-definition in Power System Security Analysis," 4th Int. Conf. Electr. Util. Deregul. Restruct. Power Technol., pp. 63-66, 2011.

[7] A. Khodaei and M. Shahidehpour, "Transmission switching in security-constrained unit commitment," IEEE Trans. Power Syst., vol. 25, no. 4, pp. 1937-1945, 2010.

[8] M. Flores, R. Romero, and J. F. Franco, "An analysis of the optimal switching problem in transmission systems," IEEE PES Innov. Smart Grid Technol. Conf. - Lat. Am. ISGT Lat. Am., vol. 2017-Janua, pp. 1-5, 2017.

[9] J. Aghaei, A. Nikoobakht, M. Mardaneh, M. Shafie-khah, and J. P. S. Catalão, "Transmission switching, demand response and energy storage systems in an innovative integrated scheme for managing the uncertainty of wind power generation," Int. J. Electr. Power Energy Syst., vol. 98, no. August 2017, pp. 72-84, 2018.

[10] A. Nikoobakht, J. Aghaei, and M. Mardaneh, "Securing highly penetrated wind energy systems using linearized transmission switching mechanism," Appl. Energy, vol. 190, pp. 1207-1220, 2017.

[11] X. Li, P. Balasubramanian, M. Sahraei-Ardakani, M. Abdi-
Khorsand, K. W. Hedman, and R. Podmore, "Real-Time Contingency Analysis with Corrective Transmission Switching," IEEE Trans. Power Syst., vol. 32, no. 4, pp. 2604-2617, 2017.

[12] G. Poyrazoglu and H. Oh, "Optimal Topology Control with Physical Power Flow Constraints and N-1 Contingency Criterion," IEEE Trans. Power Syst., vol. 30, no. 6, pp. 3063-3071, 2015.

[13] P. Escudero and D. Carrión, "Expansión de un sistema de transmisión mediante LOPF-AC," Inge Cuc, vol. 14, no. 2, pp. 116-125, 2018.

[14] M. Khanabadi, Y. Fu, and C. Liu, "Decentralized transmission line switching for congestion management of interconnected power systems," IEEE Trans. Power Syst., vol. 33, no. 6, pp. 5902-5912, 2018.

[15] D. Carrión, J. W. González, I. A. Issac, and G. J. López, "Optimal fault location in transmission lines using hybrid method," 2017 IEEE PES Innov. Smart Grid Technol. Conf. - Lat. Am. ISGT Lat. Am. 2017, pp. 1-6, 2017.

[16] S. Robak, J. Machowski, and K. Gryszpanowicz, "Contingency selection for power system stability analysis," 2017 18th Int. Sci. Conf. Electr. Power Eng., pp. 0-4, 2017.

[17] G. Papaefthymiou, J. Verboomen, and P. H. Schavemaker, "impact of Stochastic Generation in Power Systems Contingency Analysis," Int. Conf. Probabilistic Methods Appl. to Power Syst., pp. 1-6, 2006.

[18] F. Fatehi, M. Rashidinejad, and A. A. Gharaveisi, "Contingency Ranking Based on a Voltage Stability Criteria Index," 2007 Large Eng. Syst. Conf. Power Eng., pp. 142-147, 2007.

[19] M. Lu, H. Nagarajan, E. Yamangil, R. Bent, S. Backhaus, and A. Barnes, "Optimal Transmission Line Switching Under Geomagnetic Disturbances," IEEE Trans. Power Syst., vol. 33, no. 3, pp. 2539-2550, 2018.

[20] M. Peker, A. S. Kocaman, and B. Y. Kara, "Benefits of transmission switching and energy storage in power systems with high renewable energy penetration," Appl. Energy, vol. 228, no. June, pp. 1182-1197, 2018.

[21] Y. Tohidi, M. R. Hesamzadeh, R. Baldick, and D. R. Biggar, "Transmission network switching for reducing market power cost in generation sector: A Nash-equilibrium approach," Electr. Power Syst. Res., vol. 146, pp. 71-79, 2017.

[22] Q. Wang, J. P. Watson, and Y. Guan, "Two-stage robust optimization for N-k contingency-constrained unit commitment," IEEE Trans. Power Syst., vol. 28, no. 3, pp. 2366-2375, 2013.

[23] S. K. Agarwal and W. V. Torre, "Development of reliability targets for planning transmission facilities using probabilistic techniques - A utility approach," IEEE Trans. Power Syst., vol. 12, no. 2, pp. 704-709, 1997.

[24] A. Khodaei, M. Shahidehpour, and S. Kamalinia, "Transmission Switching in Expansion Planning," IEEE Trans. Power Syst., vol. 25, no. 3, pp. 1-9, 2010. 
[25] A. Gary and A. Mass, "Evaluacion de confiabilidad en sistemas electricos de distribucion," Pontificia Universidad Catóica de chile, 1994.

[26] P. Dehghanian and M. Kezunovic, "Probabilistic impact of transmission line switching on power system operating states," Proc. IEEE Power Eng. Soc. Transm. Distrib. Conf., vol. 2016-July, pp. 1-5, 2016.

[27] M. Khanabadi, H. Ghasemi, and M. Doostizadeh, "Optimal transmission switching considering voltage security and $\mathrm{N}$ 1 contingency analysis," IEEE Trans. Power Syst., vol. 28, no. 1, pp. 542-550, 2013.

[28] R. Caglar and A. Ozdemir, "Composite Electric Power System Adequacy Evaluation via Transmission Losses Based Contingency Selection Algorithm," PowerTech Budapest 99. Abstr. Rec., p. 84, 1999.

[29] G. Ayala and A. Street, "Energy and reserve scheduling with post-contingency transmission switching," Electr. Power Syst. Res., vol. 111, pp. 133-140, 2014.

[30] F. Pourahmadi, M. Jooshaki, and S. H. Hosseini, "A dynamic programming-based heuristic approach for optimal transmission switching problem with $\mathrm{N}-1$ reliability criterion," 2016 Int. Conf. Probabilistic Methods Appl. to Power Syst. PMAPS 2016 - Proc., 2016.

[31] S. A. Tabatabaei Khorram, M. Fotuhi-Firuzabad, and A. Safdarian, "Optimal transmission switching as a remedial action to enhance power system reliability," 2016 Smart Grids Conf. SGC 2016, pp. 7-12, 2017.

[32] S. Pal, S. Sen, J. Bera, and S. Sengupta, "Network modeling using optimal transmission switching," IEEE Calcutta Conf. CALCON 2017 - Proc., vol. 2018-Janua, pp. 321324, 2018.

[33] D. Sun, X. Liu, Y. Wang, B. Yang, and J. Yang, "Robust optimal power flow with transmission switching," Proc. IECON 2017 - 43rd Annu. Conf. IEEE Ind. Electron. Soc., vol. 2017-Janua, pp. 416-421, 2017.

[34] K. W. Hedman, R. P. O’Neill, E. B. Fisher, and S. S. Oren, "Optimal Transmission Switching With Contingency Analysis," IEEE Trans. power Syst., vol. 24, no. 3, pp. 1577-1586, 2009.

[35] M. Abdi-Khorsand, M. Sahraei-Ardakani, and Y. M. AlAbdullah, "Corrective Transmission Switching for N-1-1 Contingency Analysis," IEEE Trans. Power Syst., vol. 32, no. 2, pp. 1606-1615, 2017.

[36] D. Carrion and J. Gonzalez, "Optimal PMU location in electrical power systems under N-1 contingency," Proc. 3rd Int. Conf. Inf. Syst. Comput. Sci. INCISCOS 2018, no. 1, pp. 165-170, 2018.

[37] D. Carrión and J. González, "Ubicación óptima de una PMU considerando restricciones de contingencias N-1 en los sistemas eléctricos de potencia," Enfoque UTE, vol. 10, no. 1, pp. 1-12, 2019.

[38] F. H. Jufri, V. Widiputra, and J. Jung, "State-of-the-art review on power grid resilience to extreme weather events: Definitions, frameworks, quantitative assessment methodologies, and enhancement strategies," Appl. Energy, vol. 239, no. July 2018, pp. 1049-1065, 2019.

[39] Y. Khalil, R. El-Azab, M. A. Abu Adma, and S. Elmasry, "Transmission Lines Restoration Using Resilience Analysis," 2018 20th Int. Middle East Power Syst. Conf. MEPCON 2018 - Proc., pp. 249-253, 2019.

[40] Y. Lin, Z. Bie, and A. Qiu, "A review of key strategies in realizing power system resilience," Glob. Energy Interconnect., vol. 1, no. 1, pp. 70-78, 2018.

[41] Ieee, IEEE Guide for Electric Power Distribution Reliability Indices IEEE, vol. Std 1366-2. New York: IEEE, 2012.

[42] a. K. Basu, S. Chowdhury, and S. P. Chowdhury, "Role of switching devices on microgrid reliability," Univ. Power Eng. Conf. (UPEC), 2010 45th Int., no. 1, pp. 1-6, 2010.

[43] D. L. Johnston and B. K. Johnson, "Comparison of reliability indices with the effect of protection failure for an electrical to hydrogen distribution system," 41 st North Am. Power Symp. NAPS 2009, pp. 1-5, 2009.

[44] H. Lotfi, M. B. Elmi, and M. Zarif, "Reliability assessment for power grid by adding wind farm," 2nd Int. Congr. Technol. Commun. Knowledge, ICTCK 2015, no. Ictck, pp. 170-176, 2016.

[45] A. K. Rajeevan, P. V. Shouri, and U. Nair, "ARIMA modeling of wind speed for wind farm reliability analysis," 2014 Annu. Int. Conf. Emerg. Res. Areas Magn. Mach. Drives, pp. 3-7, 2014.

[46] L. Xie, L. Cheng, and Y. Gu, "Reliability assessment at day-ahead operating stage in power systems with wind generation," Proc. Annu. Hawaii Int. Conf. Syst. Sci., pp. 2245-2251, 2013.

[47] D. A. K, "Reliability Criteria for Power Systems with Inherent Generation and Transmission Deficiencies," IEEE Trans. Reliab., vol. R-30, no. 1, pp. 18-21, 1981. 